\title{
Nível de atividade física no lazer dos professores de educação física do ensino básico
}

\author{
Leisure time physical activity level of primary and secondary school physical \\ education teachers
}

\author{
Lúcio Kerber Canabarro ${ }^{1}$ \\ Marilda Borges Neutzling ${ }^{1,2}$ \\ Airton José Rombaldi ${ }^{1}$ \\ 1-PPG Educação Física - Universidade \\ Federal de Pelotas \\ 2- Departamento de Medicina Social. \\ Faculdade de Medicina. Universidade \\ Federal do Rio Grande do Sul. Porto \\ Alegre, RS, Brasil
}

\begin{abstract}
Resumo
Introdução: Estimar o nível de atividade física é importante para o planejamento de intervenções, no entanto há poucos trabalhos que investigam o comportamento de professores neste aspecto. Objetivo: verificar, através de um estudo transversal, o nível de atividade física no lazer dos professores de educação física do ensino básico que atuam na zona urbana da cidade de Pelotas - RS. Método: estudo de caráter censitário, que utilizou o IPAQ (International Physical Activity Questionnarie) longo para verificação do nível de atividade física no lazer destes profissionais. Resultados: 188 professores responderam o questionário, $61,3 \%$ do sexo feminino, 55,4\% concluíram pós-graduação e $56,4 \%$ foram classificados como ativos no lazer. O nível de atividade física no lazer, na análise ajustada, apresentou associação inversa com tabagismo $(p=0,04)$ e direta com autopercepção de saúde $(p=0,001)$. Conclusão: os professores apresentaram um nível de atividade física no lazer superior aos encontrados na população de Pelotas e não demonstraram os mesmos fatores de risco para inatividade física que a população em geral.
\end{abstract}

Palavras-chave: Professores; Educação física, Atividade física no lazer
Endereço para Correspondência

Airton José Rombaldi

Rua Luis de Camões, 625 - Bairro Areal

Pelotas - RS

CEP 96055-630

e-mail: rombaldi@brtrubo.com.br

- Recebido: 21/5/2010

- Re-submissão: 17/07/2010

$06 / 08 / 2010$

- Aceito: 11/8/2010

\section{Abstract}

Introduction: To estimate the level of physical activity is important for planning interventions, however there are few studies that investigate the teacher's behavior in this aspect. Objective: the aim of this study was to verify, through a cross-sectional study, the level of physical activity of primary school and high school physical education teachers working in the urban area of the city of Pelotas, southern Brazil. Method: a census study, using the IPAQ long to check the level of physical activity in the leisure of these professionals. Results: 188 teachers answered the questionnaire, $61.3 \%$ females, $55.4 \%$ completed post graduation studies and $56.4 \%$ were classified as active in leisure-time. The level of physical activity during leisure time, in the adjusted analysis, presented inverse association with smoking $(p=0.04)$ and direct with health's self-perception $(p=0.001)$. Conclusion: The teachers presented a level of leisure time physical activity considered higher than those found in the population of Pelotas and have not shown the same risk factors for physical inactivity when compared with the population in general.

Keywords: Teachers, Physical education, Leisure time physical activity 


\section{INTRODUÇÃO}

Grande número de evidências ${ }^{1-5}$ tem demonstrado que baixos níveis de atividade física, o hábito de fumar e a má alimentação são comportamentos responsáveis por grande número de doenças, tais como obesidade, diabetes, hipertensão, infarto, e alguns tipos de câncer. Sabe-se também que o hábito de realizar atividades físicas regulares, por no mínimo cento e cinqüenta minutos semanais em adultos, exerce um efeito protetor contra muitas destas enfermidades ${ }^{6}$.

O âmbito profissional parece também ser fator de risco para determinadas doenças. Profissionais da educação, em especial professores, parecem estar mais expostos a doenças relacionadas a problemas vocais, lesões por movimentos repetitivos, alergias e transtornos emocionais ${ }^{7-9}$. No Brasil, segundo dados do INEP, existem cerca de 1.542 .879 professores atuando no ensino básico, cerca de 60 mil somente de educação física, sendo 5000 no estado do Rio Grande do Sul ${ }^{10}$, número expressivo que carece de estudos que possam nortear políticas específicas para melhora e/ou prevenção de doenças e agravos a saúde.

No que tange ao nível de atividade física dos professores que lecionam a disciplina de educação física os dados parecem ser mais escassos, pois parece haver uma espécie de senso comum que parte do pressuposto de que o professor de uma determinada disciplina é naturalmente mais ativo, o que os exclui de determinadas investigações ${ }^{7,8}$. Entretanto, alguns trabalhos realizados na Europa com professores de educação física apresentaram níveis de atividade física superiores a seus colegas que ministram outras disciplinas como também a população em geral ${ }^{11-13}$. Conhecer assim os níveis de atividade física e fatores e grupos de risco para o sedentarismo na população de professores brasileiros é uma necessidade a fim de viabilizar intervenções específicas e com mais chances de serem eficazes ${ }^{14}$.

Tendo em vista a pequena quantidade de artigos internacionais abordando o tema ${ }^{11-13}$ e a inexistência de estudos nacionais de base populacional e conduzidos com instrumentos que possibilitem a comparação da prática de atividade física com o restante da população, o presente estudo tem como objetivo verificar, de forma transversal e com caráter censitário, o nível de atividade física no lazer dos professores de educação física do ensino básico da zona urbana da cidade de Pelotas, RS.

\section{METODOLOGIA}

Foi realizado um estudo observacional, de caráter transversal e cunho censitário. Foram contatadas todas as 112 escolas de ensino básico da zona urbana da cidade de Pelotas, RS, elegíveis para o estudo. Foi solicitado junto a Secretaria Municipal de Educação, responsável pelas escolas públicas municipais, e a $5^{\circ}$ Coordenadoria Regional de Educação, encarregada das escolas estaduais e particulares, uma autorização, devidamente protocolada para efetuar a pesquisa nas instituições de ensino. Juntamente com o pedido de autorização para efetuar a pesquisa, foi solicitada a relação de todas as escolas em funcionamento existentes na zona urbana do município, documento que serviu de base para nortear a coleta de dados.

O instrumento utilizado para avaliar o nível de atividade física foi o International Physical Activity Questionnaire (IPAQ), versão longa, composto por questões que abordam os domínios de lazer, deslocamento, atividades domésticas e trabalho. Para o presente estudo somente foi considerado o domínio do lazer em uma semana habitual, na medida em que o nível de atividade física relatada nos ambientes do trabalho e doméstico parece ser superestimado ${ }^{15-17}$, e o domínio do lazer parece ser o mais lembrado ${ }^{18,19}$. O escore foi calculado da seguinte forma: minutos por semana de caminhada + minutos por semana de atividade física moderada + (minutos por semana de atividade física vigorosa $* 2)^{20}$. Foram considerados ativos os professores que atingiram 150 minutos de atividade física semanal e insuficientemente ativos aqueles que ficaram abaixo deste valor. Dessa forma, o desfecho do presente estudo foi o nível suficiente de prática de atividade física no lazer. O nível sócio-econômico determinado segundo classificação da Associação Brasileira de Empresas de Pesquisa ${ }^{21}$. O peso e a altura foram autoreferidos.

Os questionários foram autoaplicados, sendo que $98,9 \%$ foram preenchidos no ambiente escolar. Excepcionalmente, o local de coleta foi outro ambiente de trabalho (academias) ou na residência do profissional. Foram considerados professores elegíveis todos os que estavam exercendo função docente no período de coleta de dados; profissionais que estavam trabalhando exclusivamente em funções administrativas, em desvio de função ou em licença de qualquer natureza foram excluídos. A informação do número de professores de educação física que trabalhavam na escola era fornecida pelo diretor(a) ou coordenador(a) pedagógico(a) e, em caso de dúvida, consultava-se o livro ponto. Foram consideradas recusas professores que expressaram verbalmente o desejo de não participar do estudo por duas vezes, com intervalo mínimo de uma semana. O período de coleta foi de junho a dezembro de 2009.

Contou-se com o apoio de nove acadêmicos (as) oriundos (as) dos cursos de nutrição e educação física da Universidade Federal de Pelotas, para o contato com os professores. Os acadêmicos passaram por treinamento de 12 horas. Esses auxiliares de pesquisa ajudavam na compreensão do questionário e auxiliavam em caso de dúvida sobre o preenchimento. Dez por cento dos sujeitos de pesquisa foram sorteados para o controle de qualidade o qual se deu através de contatos por telefone com os professores e por visitas as instituições escolares. Nas re-entrevistas foram aplicadas 15 questões pré-selecionadas de modo a verificar a consistência das respostas. No caso de inconsistência, as entrevistas seriam refeitas.

Todos os participantes assinaram o termo de consentimento livre e esclarecido, e a pesquisa foi aprovada pelo Comitê de Ética da Escola Superior de Educação Física da Universidade Federal de Pelotas (ESEF/UFPel) com número de protocolo 032/2009.

O banco de dados foi construído no Epi-Info 6.0, sendo realizada dupla digitação de cada questionário a fim de verificar erros de digitação. Para a análise dos dados utilizou-se os programas SPSS versão 13.0 e Stata, versão 9.0. Foi feita uma análise descritiva dos sujeitos em termos do nível de atividade física no lazer e das variáveis socioeconômica, demográficas, comportamentais, de saúde e de trabalho. A análise bruta entre o desfecho e as variáveis independentes foi testada através de testes de qui-quadrado para diferença de proporção e qui-quadrado para tendência linear. $A$ análise multivariável foi conduzida através de regressão de Poisson. Foram mantidas na análise todas aquelas variáveis que apresentaram valor $\mathrm{p} \leq 0,2$ na análise bruta para controle de confusão. O nível de significância estabelecido foi de $5 \%$. Na análise multivariável todas as variáveis foram colocadas ao mesmo tempo e a seguir realizou-se uma seleção para trás. 


\section{RESULTADOS}

O número de professores de educação física atuando nas redes pública e privada do ensino básico na zona urbana da cidade Pelotas, RS era de 212. Destes, 188 responderam ao questionário, fato que representa uma taxa de respostas de $88,7 \%$. A variável independente com maior número de valores ignorados foi "Jornada de trabalho semanal" com 18 indivíduos não informando suas cargas horárias semanais. No controle de qualidade não se detectou nenhuma inconsistência nas entrevistas.

Na tabela 1 verifica-se que a maioria dos indivíduos era do sexo feminino (61,7\%), tinha entre 31 a 50 anos (70,3\%), pertencia ao nível socioeconômico $B(66,0 \%)$, nunca fumaram $(78,5 \%)$, percebiam sua saúde como excelente e muito boa $(62,6 \%)$ e estavam eutróficos $(60,9 \%)$.

A maioria dos respondentes (97,3\%), apresentava graduação concluída em educação física e 55,4\% destes possuíam titulação de pós-graduação, dos quais $87,1 \%$ de especialização, 11,9\% de mestrado e 1,0\% de doutorado. A maioria dos professores apresentava até 15 anos de trabalho no ambiente escolar $(61,7 \%)$, não exercia outra atividade remunerada $(69,7 \%)$, exercia carga horária semanal total de até $40 \mathrm{~h}$ $(75,4 \%)$, trabalhava na rede pública de ensino (78\%) e era ativo no lazer (56,4\%) (tabela 2$)$.

A análise bruta não mostrou associação significativa do desfecho (nível suficiente de atividade física no lazer) com as variáveis sexo, idade e nível socioeconômico, que se mantiveram sem significância na análise ajustada. As variáveis ta- bagismo e autopercepção de saúde apresentaram associação significativa com o desfecho na análise bruta e se mantiveram significantes na análise ajustada $(p=0,04 ; p=0,001$, respectivamente). O IMC não apresentou qualquer relação com a prática de atividade física no lazer tanto na análise bruta como na ajustada (tabela 3).

Possuir pós-graduação, anos de serviço em escolas, ter emprego fora do ambiente escolar, a jornada de trabalho e a rede de ensino em que o professor atua não se associaram significativamente com o desfecho (tabela 4).

\section{DISCUSSÃO}

Um aspecto a ser destacado nesse estudo é seu caráter censitário e o baixo índice de perdas e recusas (11,3\%). Por outro lado, é necessário considerar que as diferenças entre os métodos e instrumentos para avaliar nível de atividade física prejudicam a comparação com outros estudos.

O nível suficiente de atividade física no lazer foi encontrado em 56,4\% dos professores de educação física. Essa freqüência foi maior do que aquela verificada na população brasileira em geral $(14,8 \%)^{22}$ e na população de Pelotas/RS, onde apenas $30,0 \%$ dos indivíduos eram ativos no lazer ${ }^{23}$. Além disso, estudos internacionais têm concordado que o nível de atividade física entre professores de educação física é maior do que aquele observado em outros professores ou outros profissionais. Na Finlândia em 2007, Rintala et al. constataram que $30 \%$ das professoras de educação física, eram ativas no lazer comparado com apenas $10 \%$ das professoras de idiomas ${ }^{11}$.

\begin{tabular}{ccc}
\hline \multicolumn{1}{c}{ Variáveis } & \multicolumn{2}{c}{ Total } \\
\cline { 2 - 3 } Sexo & $\mathrm{n}$ & $\%$ \\
Masculino & 72 & 38,7 \\
Feminino & 114 & 61,3 \\
Idade (anos) & & \\
20-30 & 33 & 17,5 \\
$31-40$ & 58 & 30,9 \\
$41-50$ & 74 & 39,4 \\
Z51 & 23 & 12,2 \\
Nível socioeconômico & & \\
A & 59 & 31,4 \\
B & 124 & 66,0 \\
C & 5 & 2,6 \\
Tabagismo & & \\
Fumante & 16 & 8,6 \\
Ex-fumante & 24 & 12,9 \\
Nunca fumou & 146 & 78,5 \\
Autopercepção de saúde & & \\
Excelente & 35 & 18,7 \\
Muito boa & 82 & 43,9 \\
Boa & 62 & 33,2 \\
Regular/Ruim & 8 & 4,2 \\
IMC (Kg/m & & \\
Eutrófico & 112 & 60,9 \\
Sobrepeso & 58 & 31,5 \\
Obesidade & 14 & 7,6 \\
\hline
\end{tabular}




\begin{tabular}{|c|c|c|}
\hline \multirow{2}{*}{ Variáveis } & \multicolumn{2}{|c|}{ Total } \\
\hline & $\mathrm{n}$ & $\%$ \\
\hline \multicolumn{3}{|l|}{ Graduado em Educação Física } \\
\hline Sim & 183 & 97,3 \\
\hline Não & 5 & 2,7 \\
\hline \multicolumn{3}{|l|}{ Possui pós-graduação } \\
\hline Sim & 103 & 55,4 \\
\hline Não & 83 & 44,6 \\
\hline \multicolumn{3}{|l|}{ Anos de serviço em escola (anos) } \\
\hline Até 5 & 47 & 25,0 \\
\hline $6-15$ & 69 & 36,7 \\
\hline $16-25$ & 48 & 25,5 \\
\hline$\geq 26$ & 24 & 12,8 \\
\hline \multicolumn{3}{|l|}{ Trabalho exercido fora da escola } \\
\hline Sim & 57 & 30,3 \\
\hline Não & 131 & 69,7 \\
\hline \multicolumn{3}{|l|}{ Jornada de trabalho semanal (horas) } \\
\hline Até 20 & 42 & 24,6 \\
\hline $21-30$ & 17 & 9,9 \\
\hline $30-40$ & 70 & 40,9 \\
\hline$\geq 41$ & 42 & 24,6 \\
\hline \multicolumn{3}{|l|}{$\begin{array}{l}\text { Rede de ensino em que atua } \\
\text { Pública }\end{array}$} \\
\hline Municipal & 66 & 35,5 \\
\hline Estadual & 30 & 16,1 \\
\hline Municipal e estadual & 35 & 18,8 \\
\hline Federal & 14 & 7,6 \\
\hline Particular & 19 & 10,2 \\
\hline Particular e rede pública & 22 & 11,8 \\
\hline \multicolumn{3}{|l|}{ Nível de atividade física no lazer } \\
\hline Insuficientemente ativo & 81 & 43,6 \\
\hline Ativo & 105 & 56,4 \\
\hline
\end{tabular}

Achados semelhantes foram relatados por Pihl et al. quando compararam professores de educação física com docentes de outras disciplinas da mesma idade na Estônia; os autores encontraram que $59,3 \%$ dos primeiros eram ativos no lazer contra $11,7 \%$ do grupo dos demais professores ${ }^{13}$. Entre profissionais de saúde das regiões nordeste e sul do Brasil o nível de atividade física foi de $72,5 \%$ de ativos ${ }^{14}$ e entre enfermeiras mexicanas $21 \%{ }^{24}$. É importante salientar, no entanto, que o desfecho de nosso estudo considerou apenas o domínio lazer, enquanto Siqueira e colaboradores ${ }^{14}$ verificaram todos os domínios da atividade física (lazer, deslocamento, afazeres domésticos e trabalho), o que dificulta a comparação dos resultados dada a reconhecida superestimativa de prática de atividade física relatada especialmente no domínio doméstico ${ }^{15-17}$.

No presente estudo o nível de atividade física foi maior entre indivíduos não fumantes e com melhor percepção sobre sua saúde. Resultados similares foram encontrados em outros estudos ${ }^{14,25}$. Em educadores físicos da Croácia, 70\% dos professores não fumavam; essa freqüência era mais elevada que aquela verificada na população croata em geral ${ }^{26}$. No estudo desenvolvido com profissionais de saúde nas regiões sul e nordeste do Brasil maiores níveis de atividade física foram encontrados entre indivíduos não fumantes e naqueles que consideravam sua saúde muito boa ou excelente ${ }^{14}$. No mesmo sentido resultados de estudo populacional realizado em Pelotas, em 2003, com adultos ${ }^{25}$ revelaram associação positiva entre melhores percepções de saúde e nível de atividade física. Ressalta-se ainda que apenas $8,6 \%$ dos professores do presente estudo eram fumantes, valor inferior a prevalência nacional da população em geral $(23 \%)^{27}$. Recentes estudos tem analisado e discutido o fato de que hábitos de vida saudáveis aparecem agregados ${ }^{28,29}$, havendo indícios de que pessoas que ingerem mais frutas e verduras sejam mais ativas ${ }^{29}$. Ao contrário, morbidades como a hipertensão, aparecerem associadas aos fatores de risco sobrepeso, inatividade física e avanço da idade, o que torna o incentivo a prática de atividade física ainda mais premente ${ }^{30}$.

Observou-se no presente estudo que as variáveis sexo, idade, nível socioeconômico, IMC, formação acadêmica, jornada de trabalho e tempo de serviço, não estiveram associadas com o desfecho nível de atividade física no lazer. Nossos resultados, provavelmente em função do conhecimento profissional, contrariam estudos populacionais, os quais indicam que pertencer ao sexo masculino representa fator de proteção à inatividade física no lazer ${ }^{22,23,31}$.

Com relação a idade, muitos autores $22,23,25,30$ mostram que o nível de atividade física parece declinar com o avanço da idade. No entanto estudo realizado na Estônia em 2002 com 


\begin{tabular}{|c|c|c|c|c|c|}
\hline \multirow[t]{2}{*}{ Variáveis } & \multicolumn{2}{|c|}{$\begin{array}{l}\text { Atividade } \\
\text { física no } \\
\text { lazer }\end{array}$} & \multirow[t]{2}{*}{$\begin{array}{l}\text { Razões de } \\
\text { Prevalência } \\
\quad\left(\mathrm{IC}_{95 \%}\right)\end{array}$} & \multirow{2}{*}{$\begin{array}{l}\text { Razões de } \\
\text { Prevalência } \\
\text { ajustadas } \\
\left(\mathrm{IC}_{95 \%}\right)^{\#}\end{array}$} & \multirow[t]{2}{*}{ Valor $\mathrm{p}$} \\
\hline & $\mathrm{n}$ & $\%$ & & & \\
\hline Sexo & & & & & $0,2 *$ \\
\hline Masculino & 47 & 65,3 & 1,0 & 1,0 & \\
\hline Feminino & 58 & 50,9 & $0,8(0,6-1,0)$ & $0,8(0,6-1,1)$ & \\
\hline Idade (anos) & & & & & $0,4 * *$ \\
\hline $20-30$ & 22 & 66,7 & $1,5(0,9-2,5)$ & $0,9(0,5-1,6)$ & \\
\hline $31-40$ & 31 & 53,5 & $1,2(0,7-2,0)$ & $1,0(0,6-1,6)$ & \\
\hline $41-50$ & 42 & 57,5 & $1,3(0,8-2,1)$ & $1,1(0,7-1,8)$ & \\
\hline$\geq 51$ & 10 & 45,5 & 1,0 & 1,0 & \\
\hline Nível socioeconômico & & & & & $0,9 *$ \\
\hline A & 31 & 53,5 & 1,0 & 1,0 & \\
\hline $\mathrm{B} / \mathrm{C}$ & 74 & 57,8 & $1,1(0,8-1,4)$ & $1,1(0,9-1,5)$ & \\
\hline Tabagismo & & & & & $0,04 *$ \\
\hline Fumante & 4 & 25,0 & 1,0 & 1,0 & \\
\hline Ex-fumante & 13 & 56,5 & $2,3(0,9-5,7)$ & $2,3(1,1-5,0)$ & \\
\hline Nunca fumou & 87 & 60,0 & $2,4(1,0-5,8)$ & $2,3(1,0-5,0)$ & \\
\hline Autopercepção de saúde & & & & & $0,001 * *$ \\
\hline Excelente & 26 & 76,4 & $3,1(0,9-10,0)$ & $3,0(0,9-10,0)$ & \\
\hline Muito boa & 50 & 60,1 & $2,4(0,7-8,2)$ & $2,5(0,8-8,2)$ & \\
\hline Boa & 26 & 42,6 & $1,7(0,5-5,9)$ & $1,8(0,5-5,9)$ & \\
\hline Regular/Ruim & 2 & 25,0 & 1,0 & 1,0 & \\
\hline $\operatorname{IMC}\left(\mathrm{Kg} / \mathrm{m}^{2}\right)$ & & & & & $0,6^{*}$ \\
\hline Eutrófico & 64 & 57,1 & 1,0 & 1,0 & \\
\hline Sobrepeso & 35 & 61,4 & $1,1(0,8-1,4)$ & $1,0(0,8-1,3)$ & \\
\hline Obesidade & 4 & 28,6 & $0,5(0,2-1,2)$ & $0,7(0,3-1,7)$ & \\
\hline
\end{tabular}

* Teste do qui-quadrado para heterogeneidade

** Teste de Wald para tendência linear

\# Todas as variáveis foram ajustadas entre si.

professores de educação física com idade entre 51 e 72 anos comparados a professores de outras disciplinas da mesma faixa etária mostrou que os primeiros são significativamente mais ativos no lazer, independente da idade ${ }^{13}$. Uma possível explicação para a ausência de associação entre idade dos professores e nível de atividade física no presente estudo poderia ser o conhecimento sobre os benefícios para a saúde e a afinidade com a prática regular de atividade física.

No que se refere ao nível socioeconômico verificou-se que a grande maioria $(97,4 \%)$ dos docentes, segundo a $A B E P$, pertenciam as classes A e B. Essa homogeneidade de classificação pode ter contribuído para a ausência de associação entre as variáveis e o desfecho. Importante destacar, no entanto, que o fato de grande parte dos professores pertencerem as classes A e B pode também ter contribuído para o maior nível de atividade física no lazer do que a população em geral. Outros estudos revelam que indivíduos com maior nível socioeconômico são mais ativos no lazer ${ }^{22,23}$. Autores que analisaram as barreiras para a prática de atividades físicas, verificaram que a falta de dinheiro é uma das barreiras mais fortes à pratica de atividade física no lazer, o que também auxilia no entendimento da relação entre essas variávei ${ }^{28,32}$.

No que diz respeito a variável estado nutricional (IMC), embora não sido encontrada associação significativa entre
IMC e nível de atividade física é importante salientar que as prevalências de excesso peso $(39,1 \%)$ encontradas no presente estudo eram bem inferiores aquelas verificadas na população em geral $51,3 \%^{32}$. Esses resultados sugerem que os profissionais de educação física, por serem mais ativos no lazer que a população em geral, seriam também mais eutróficos. Essa menor prevalência de sobrepeso entre os professores de educação física foi observada também em um outro estudo realizado na Suécia em $2000^{12}$.

A hipótese de que professores com jornadas de trabalho maiores e mais tempo de serviço seriam mais sedentários não foram comprovadas, haja vista que a análise ajustada revelou valores $p$ de 0,7 e 0,5 , respectivamente. Resultados semelhantes foram encontrados por Both et al. ${ }^{33} \mathrm{com}$ professores de educação física do estado de Santa Catarina, onde a jornada de trabalho e o tempo de serviço não apresentaram associação significativa com a prática de atividades físicas. Por outro lado, Lemos ${ }^{34} \mathrm{em}$ estudo realizado com professores de educação física da rede estadual de ensino do Rio Grande do Sul, verificou que profissionais com jornadas de trabalho de 40 horas ou mais relatavam melhores hábitos quanto a prática de atividades físicas. Cabe destacar, no entanto que o instrumento utilizado nesse estudo verificou qualidade de vida como um todo, distinto do empregado na presente pesquisa ${ }^{12,32}$. No 


\begin{tabular}{|c|c|c|c|c|c|}
\hline \multirow[t]{2}{*}{ Variáveis } & \multicolumn{2}{|c|}{$\begin{array}{l}\text { Atividade física } \\
\text { no lazer }\end{array}$} & \multirow{2}{*}{$\begin{array}{l}\text { Razões de } \\
\text { Prevalência } \\
\quad\left(\mathrm{IC}_{95 \%}\right)\end{array}$} & \multirow{2}{*}{$\begin{array}{l}\text { Razões de } \\
\text { Prevalência } \\
\text { ajustadas } \\
\left(\mathrm{IC}_{95 \%}\right)^{\#}\end{array}$} & \multirow[t]{2}{*}{ Valor $\mathrm{p}$} \\
\hline & $\mathrm{n}$ & $\%$ & & & \\
\hline Possui pós-graduação & & & & & $0,4 *$ \\
\hline Sim & 53 & 52,0 & 1,0 & 1,0 & \\
\hline Não & 51 & 62,2 & $1,2(0,9-1,5)$ & $1,1(0,9-1,4)$ & \\
\hline $\begin{array}{l}\text { Anos de serviço em escola } \\
\text { (anos) }\end{array}$ & & & & & $0,5 * *$ \\
\hline Até 5 & 28 & 59,6 & 1,0 & 1,0 & \\
\hline $6-15$ & 41 & 59,4 & $1,0(0,7-1,4)$ & $1,2(0,8-1,7)$ & \\
\hline $16-25$ & 29 & 61,7 & $1,0(0,7-1,4)$ & $1,2(0,7-1,9)$ & \\
\hline$\geq 26$ & 7 & 30,4 & $0,5(0,3-1,0)$ & $0,6(0,3-1,5)$ & \\
\hline $\begin{array}{l}\text { Trabalho exercido fora da } \\
\text { escola }\end{array}$ & & & & & $0,1^{*}$ \\
\hline Sim & 39 & 68,4 & $0,7(0,6-1,0)$ & $0,8(0,7-1,1)$ & \\
\hline Não & 66 & 51,2 & 1,0 & 1,0 & \\
\hline $\begin{array}{l}\text { Jornada de trabalho semanal } \\
\text { (horas) }\end{array}$ & & & & & $0,7 * *$ \\
\hline Até 20 & 26 & 61,9 & $1,2(0,8-1,7)$ & $0,9(0,6-1,4)$ & \\
\hline $21-30$ & 12 & 70,6 & $1,3(0,8-2,0)$ & $1,2(0,8-1,8)$ & \\
\hline $31-40$ & 41 & 58,6 & $1,1(0,8-1,5)$ & $1,0(0,7-1,4)$ & \\
\hline$\geq 41$ & 22 & 52,4 & 1,0 & 1,0 & \\
\hline Rede de ensino em que atua & & & & & \\
\hline Pública & & & & & $0,9 * *$ \\
\hline Municipal & 39 & 60,9 & 1,0 & 1,0 & \\
\hline Estadual & 17 & 56,7 & $0,9(0,6-1,3)$ & $1,1(0,7-1,5)$ & \\
\hline Municipal e estadual & 15 & 42,9 & $0,7(0,5-1,1)$ & $0,9(0,5-1,3)$ & \\
\hline Federal & 8 & 57,1 & $0,9(0,6-1,5)$ & $0,7(0,4-1,3)$ & \\
\hline Particular & 12 & 63,2 & $1,0(0,7-1,5)$ & $1,0(0,7-1,4)$ & \\
\hline Particular e Pública & 13 & 59,1 & $1,0(0,6-1,4)$ & $1,0(0,7-1,6)$ & \\
\hline
\end{tabular}

* Teste do qui-quadrado para heterogeneidade

** Teste de Wald para tendência linear

\# Todas as variáveis foram ajustadas entre si.

mesmo sentido Siqueira e colaboradores verificaram que profissionais com jornadas de até 20 horas nos postos de saúde eram mais ativos ${ }^{14}$.

Conclui-se que os professores de educação física da zona urbana da cidade de Pelotas, apresentam níveis de atividade física no lazer superiores, tanto a população brasileira das capitais como aos habitantes da cidade de Pelotas/RS, o que provavelmente se deve a natureza de sua ocupação.

Finalmente, é importante considerar que embora mais da metade dos docentes entrevistados faça atividade física regularmente no lazer (achado importante uma vez que é o dobro da frequência encontrada na população em geral), considerável parcela dos professores ainda é inativa no lazer. Estudos posteriores são necessários para compreender mais detalhadamente o comportamento dessa categoria profissional com o objetivo de se incentivar, através de políticas públicas, o aumento da prevalência de atividade física suficiente no lazer entre professores de educação física.

\section{Contribuição dos Autores}

Lúcio Kerber Canabarro e Airton José Rombaldi realiza- ram a revisão de literatura, conduziram as análises, confecção das tabelas, interpretação, escrita dos resultados e discussão. Marilda Borges Neutzling supervisionou o trabalho de campo e ajudou na escrita do artigo, em todas as fases.

\section{REFERÊNCIAS BIBLIOGRÁFICAS}

1. Eyre $\mathrm{H}$, Kahn R, Robertson RM, et al. Preventing cancer, cardiovascular disease, and diabetes: a common agenda for the American Cancer Society, the American Diabetes Association, and the American Heart Association. Circulation. 2004;109(25):3244-55.

2. Bassuk SS, Manson JE. Epidemiological evidence for the role of physical activity in reducing risk of type 2 diabetes and cardiovascular disease. J Appl Physiol. 2005;99(3):1193-204

3. Sofi F, Capalbo A, Cesari F, Abbate R, Gensini GF. Physical activity during leisure time and primary prevention of coronary heart disease: an updated metaanalysis of cohort studies. Eur J Cardiovasc Prev Rehabil. 2008;15(3):247-57.

4. Hackshaw AK, Law MR, Wald NJ. The accumulated evidence on lung cancer and environmental tobacco smoke. BMJ. 1997;315(7114):980-8.

5. Critchley JA, Unal B. Health effects associated with smokeless tobacco: a systematic review. Thorax. 2003;58(5):435-43.

6. Haskell WL, Lee IM, Pate RR, et al. Physical activity and public health: updated recommendation for adults from the American College of Sports Medicine and the American Heart Association. Med Sci Sports Exerc. 2007;39(8):142334.

7. Delcor NS, Araujo TM, Reis EJ, et al. Labor and health conditions of private 
school teachers in Vitória da Conquista, Bahia, Brazil. Cad Saude Publica. 2004;20(1):187-96.

8. Gasparin SM. Prevalência de transtornos mentais comuns em professores da rede municipal de Belo Horizonte, Minas Gerais, Brasil. Cad Saude Publica. 2006;22(12):2679 - 91.

9. Porto LAR, Costa I, Moura J, et al. Doenças ocupacionais em professores atendidos pelo centro de estudos da saúde do trabalhador. Rev Baiana Saude Publica. 2004;28(1)33 - 49.

10. MEC. Censo dos profissionais do magistério da educação básica, 2003. 2006.

11. Rintala P, Pukkala E, Laara E, Vihko V. Physical activity and breast cancer risk among female physical education and language teachers: a 34-year follow-up. Int J Cancer. 2003;107(2):268-70.

12. Sandmark H. Musculoskeletal dysfunction in physical education teachers. Occup Environ Med. 2000;57(10):673-7.

13. Pihl E, Matsin T, Jurimae T. Physical activity, musculoskeletal disorders and cardiovascular risk factors in male physical education teachers. J Sports Med Phys Fitness. 2002;42(4):466-71.

14. Siqueira FC, Nahas MV, Facchini LA, et al. Physical activity among health professionals from south and northeast Brazil. Cad Saude Publica. 2009;25(9):191728.

15. Lawlor DA, Taylor M, Bedford C, Ebrahim S. Is housework good for health? Levels of physical activity and factors associated with activity in elderly women. Results from the British Women's Heart and Health Study. J Epidemiol Community Health. 2002;56(6):473-8.

16. Stamatakis E, Hamer M, Lawlor DA. Physical activity, mortality, and cardiovascular disease: is domestic physical activity beneficial? The Scottish Health Survey -- 1995, 1998, and 2003. Am J Epidemiol. 2009;169(10):1191-200.

17. Stamatakis $E$, Hillsdon $M$, Primatesta P. Domestic physical activity in relationship to multiple CVD risk factors. Am J Prev Med. 2007;32(4):320-7.

18. Teychenne M, Ball K, Salmon J. Associations between physical activity and depressive symptoms in women. Int J Behav Nutr Phys Act. 2008;5:27.

19. Timperio A, Salmon J, Crawford D. Validity and reliability of a physical activity recall instrument among overweight and non-overweight men and women. J Sci Med Sport. 2003;6(4):477-91.

20. US Department of Health and Human Services. 2008 Physical Activity Guidelines for Americans. Disponível em: http://www.health.gov/paguidelines/pdf/ paguide.pdf. Acessado em 29 de setembro de 2009.

21. Associação Brasileira de Empresas de Pesquisa. Critério de classificação econômica Brasil - 2009. http://www.abep.org/novo/CMS/Utils/FileGenerate. ashx?id=46.

22. Florindo AA, Hallal PC, de Moura EC, Malta DC. Practice of physical activities and associated factors in adults, Brazil, 2006. Rev Saude Publica. 2009;43 Suppl 2:65-73.

23. Dias-da-Costa JS, Hallal PC, Wells JC, et al. Epidemiology of leisure-time physical activity: a population-based study in southern Brazil. Cad Saude Publica. 2005;21(1):275-82.

24. Venegas OU, Llerenas TC, Aguayo GA, et al. Physical activity and inactivity among female health service workers. Ginecol Obstet Mex. 2006;74(9):471-5.

25. Hallal PC, Victora CG, Wells JC, Lima RC. Physical inactivity: prevalence and associated variables in Brazilian adults. Med Sci Sports Exerc. 2003;35(11):1894900.

26. Misigoj-Durakovic M, Durakovic Z, Ruzic L, Findak V. Gender differences in cardiovascular diseases risk for physical education teachers. Coll Antropol 2004:28(suppl 2):251-7.

27. Azevedo e Silva G, Valente JG, de Almeida LM, de Moura EC, Malta DC. Tobacco smoking and level of education in Brazil, 2006. Rev Saude Publica. 2009;43 Suppl 2:48-56.

28. Reichert FF, Barros AJ, Domingues MR, Hallal PC. The role of perceived personal barriers to engagement in leisure-time physical activity. Am J Public Health. 2007;97(3):515-9.

29. Neutzling MB, Rombaldi AJ, Azevedo MR, Hallal PC. Factors associated with fruit and vegetable intake among adults in a southern Brazilian city. Cad Saude Publica. 2009;25(11):2365-74.

30. Capilheira MF, Santos IS, Azevedo MR, et al. Risk factors for chronic non-communicable diseases and the CARMEN initiative: a population based study in the south of Brazil. Cad Saude Publica. 2008;24 (12):2767-74

31. Pitanga FJ, Lessa I. Prevalence and variables associated with leisure-time sedentary lifestyle in adults. Cad Saude Publica. 2005;21(3):870-7.

32. Peixoto M, Benicio MJ. The relationship between body mass index and lifestyle in a brazilian adult population: a cross- sectional survey. Cad Saude Publica. 200723 (11) $2694-704$.

33. Both J, Nascimento J, Borgatto A. Percepção da qualidade de vida no trabalho e perfil do estilo de vida dos docentes de educação física no estado de Santa Catarina. Rev Edu Fis/UEM. 2008;19(3):377-89.

34. Lemos C. Qualidade de vida na carreira profissional de professores de educação física do magistério público estadual/RS. Florianópolis: Universidade Federal de Santa Catarina; Dissertação de mestrado, 2007 